\title{
Análisis de tratamiento de la otitis media crónica simple con miringoplastía en el Hospital Barros Luco Trudeau
}

\section{Analysis of the treatment of simple chronic otitis media with myringoplasty at Barros Luco Trudeau Hospital}

\author{
Rodrigo Pineda D. ${ }^{1}$, Michelle Albornoz P. ${ }^{2}$, Ángela Chuang C. ${ }^{1}$, \\ Fernanda Silva R. ${ }^{1}$, Andrés Nicolas N. ${ }^{1}$, Jaime Osorio M. ${ }^{1,3}$
}

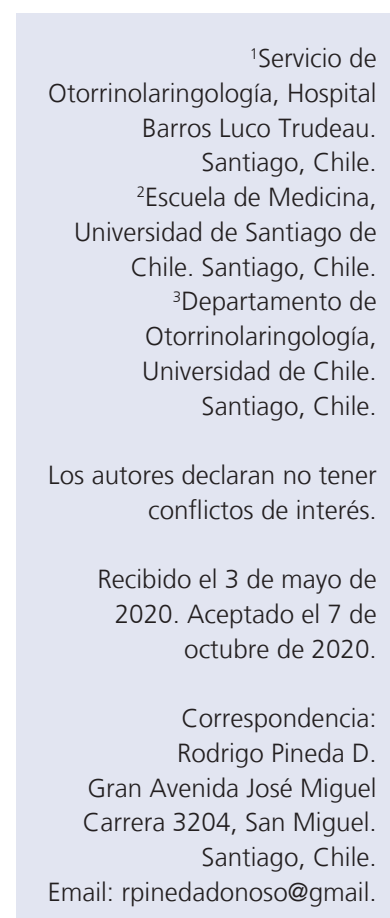

com

\section{Resumen}

Introducción: La otitis media crónica simple (OMC) es una patología común en nuestra población y hasta la fecha no queda bien claro cuál técnica y material de injerto da mejores resultados. Objetivo: Determinar si existen diferencias en los resultados anatómicos y funcionales al utilizar fascia de músculo temporal (FMT) versus injerto de cartílago de trago (CT) en los pacientes con OMC en que se les realizó miringoplastía en el Hospital Barros Luco Trudeau. Material y Método: Estudio de cohorte no concurrente de datos obtenidos de la revisión de fichas clínicas. Resultados: De 227 fichas, 154 cumplieron criterios de inclusión. En 102 pacientes (66\%) se utilizó FMT y en 52 pacientes (34\%) CT. Con FMT 38 presentaron reperforación (37\%) y 41 presentaron un éxito funcional (40\%). Con CT 18 presentaron reperforación (35\%) y 22 presentaron un éxito funcional (42\%). 38 pacientes presentaban antecedente de tabaquismo activo y de ellos 53\% presentaron reperforación, mientras que de los sin antecedentes de tabaquismo solo un $31 \%$, siendo esta diferencia estadísticamente significativa $(\mathrm{p}<0,05)$. Conclusión: No se obtuvieron diferencias estadísticamente significativas entre los resultados anatómicos y funcionales comparando el uso de injerto FMT y CT para el tratamiento quirúrgico de la OMC simple con miringoplastía.

Palabras clave: Otitis media crónica, miringoplastía, fascia músculo temporal, injerto cartílago de trago.

\footnotetext{
Abstract

Introduction: Simple chronic otitis media (COM) is a common pathology in our population, and it is currently unclear, which grafts technique and material gives the best results. Aim: To determine if there are differences in the anatomical and functional results, when using temporal muscle fascia (FMT) or tragus cartilage graft (CT) in patients with COM who underwent myringoplasty at the Barros Luco Trudeau Hospital. Material and Method: Retrospective analytical cohort study of data obtained from clinical records. Results: Of 227 medical records, 154 met inclusion criteria. FMT was used in 102 patients (66\%) and CT in 52 patients (34\%). With FMT, 38 had reperforation (37\%) and 41 had functional success (40\%). With CT 18 had reperforation (35\%) and 22 had functional success (42\%). 38 patients had a history of active smoking and 53\% of them presented reperforation, while of those without a history of smoking only 31\%, this difference being statistically significant $(p<0,05)$. Conclusion: No statistically significant differences were obtained when analyzing the anatomical and functional results comparing the use of FMT and CT graft, for the simple surgical treatment of COM with myringoplasty.

Keywords: Chronic otitis media, myringoplasty, temporal muscle fascia, tragus cartilage graft.
} 


\section{Introducción}

La otitis media crónica (OMC) es un problema común en nuestra población que puede causar hipoacusia, infecciones recurrentes del oído y, potencialmente, complicaciones meningoencefálicas. La OMC se define como la presencia de una perforación timpánica por más de tres meses siendo de etología multifactorial. Su presentación clínica se caracteriza por otorrea intermitente con una perforación timpánica que tiende a persistir y habitualmente se acompaña de hipoacusia ${ }^{1}$.

El tratamiento de la otitis media crónica simple es la miringoplastía, cirugía destinada a restaurar la integridad de la membrana timpánica, sin revisión de la cadena de huesillos, evitando la posibilidad de secuelas o complicaciones otológicas e intracraneanas. Además de mejorar el mecanismo de transmisión del sonido como objetivo secundario. Los métodos utilizados en esta intervención y las características del paciente al momento de la cirugía determinan el éxito del tratamiento ${ }^{1}$.

La miringoplastía ha sido uno de los hitos en la otología, desde los primeros intentos quirúrgicos en el campo de la cirugía del oído. Fue descrita por primera vez como miringoplastía por Berthold en 1878, quien reparó exitosamente perforaciones con un delgado injerto de piel. Durante los siguientes años el interés por este procedimiento pareció haberse perdido, sin embargo, a consecuencia del uso de microscopios, Zollner y Wullstein iniciaron las nuevas técnicas de timpanoplastía en la reconstrucción de oídos dañados por otitis media².

Respecto a los materiales del injerto se utilizan: grasa, cartílago (con o sin pericondrio), fascia del músculo temporal o una combinación de éstos. A su vez, el injerto utilizado puede instalarse en forma medial (técnica Austin), lateral (técnica House), en isla, empalizada o en mariposa ${ }^{3-7}$. La técnica medial clásicamente se ha considerado para perforaciones pequeñas, con buen margen anterior y de fácil visualización; mientras que la variante lateral es preferida en perforaciones totales o subtotales en las que no exista certeza de un adecuado margen anterior. Por su parte, para la selección del injerto se consideran las características de resistencia de la membrana timpánica del paciente, la capacidad ventila- toria del oído medio y los intentos fallidos de cierre de perforación. Todas estas variables pueden afectar los resultados quirúrgicos y funcionales de la intervención ${ }^{3}$.

El fracaso anatómico de la cirugía se define como la aparición de una nueva perforación del injerto después del cierre completo y se estima una prevalencia del $8 \%$ con cartílago y $18 \%$ con fascia ${ }^{2}$. Por otra parte, se define éxito funcional como cierre de gap óseo-aéreo sobre 10 a 15 decibel (dB) comparando audiometrías preoperatorias y posoperatorias ${ }^{2,3}$. El resultado entre el uso de fascia y cartílago es controversial en la literatura, sin existir una clara diferencia de éxito funcional ${ }^{2}$.

Existen múltiples factores que influyen en estos resultados, jugando un papel importante la edad del paciente, tipo de injerto, técnica quirúrgica y experiencia del cirujano ${ }^{4}$. Otra de las variables que se describen en la literatura es el tabaquismo. Sus efectos en el oído medio y la trompa de Eustaquio se pueden clasificar en locales, regionales y sistémicos. Los efectos locales influyen en el estado y la función del sistema mucociliar y el suministro vascular al injerto, los efectos regionales son los que resultan de la obstrucción de la trompa de Eustaquio y los efectos sistémicos son sus propiedades quimioalérgicas o inmunosupresoras, que pueden conducir a infecciones recurrentes ${ }^{4}$. Una combinación de estos factores dará como resultado un oído supurativo crónico que tiene una menor probabilidad de un resultado quirúrgico exitoso a corto y largo plazo en comparación con el de un no fumador ${ }^{5}$.

\section{Objetivo}

El objetivo principal de este estudio es determinar si existen diferencias en los resultados anatómicos y funcionales, al utilizar fascia de músculo temporal versus injerto de cartílago de trago con o sin pericondrio en los pacientes con OMC, a los que se les realizó miringoplastía en el Hospital Barros Luco Trudeau. Por otra parte, como objetivos secundarios se analizarán los resultados anatómicos en pacientes con tabaquismo activo, según las diferentes técnicas de colocación del injerto y según el tamaño de la perforación. 


\section{Material y Método}

Se realizó un estudio de cohorte no concurrente, evaluando 227 fichas clínicas de pacientes operados de miringoplastía en el Hospital Barros Luco Trudeau entre los años 2010 a 2017. Se incluyeron pacientes hombres y mujeres sobre 7 años de edad con diagnóstico de otitis media crónica simple que presentaran audiometría previa y posterior a cirugía y sin antecedentes de otorrea por lo menos 3 meses previos a cirugía. Se excluyeron a pacientes con antecedentes de miringoplastía previa, otitis media crónica colesteatomatosa, otitis media crónica fibroadhesiva y antecedentes de audiometría previa a cirugía con un gap óseo-aéreo mayor a $30 \mathrm{~dB}$.

Con el fin de dar respuesta al objetivo principalprincipal se analizó las siguientes variables primarias: Resualtado anatómico, buscando el antecedente de indemnidad o reperforación del injerto. Resultado funcional, considerando como éxito funcional diminución del gap óseo-aéreo mayor o igual a $10 \mathrm{~dB}$. También se incluyeron variables secundarias tales como: Sexo, edad, antecedentes de tabaquismo,

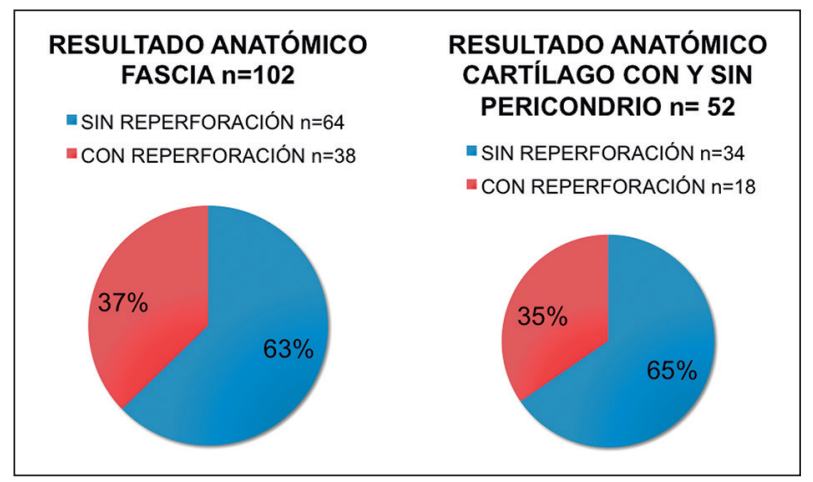

Figura 1. Resultado anatómico según tipo de injerto. ${ }^{*} \mathrm{Chi}^{2}: \mathrm{p}>0,05$.

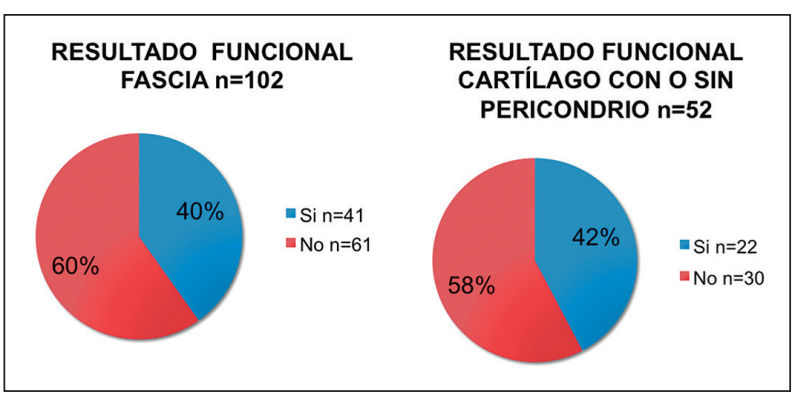

Figura 2. Resultado funcional según tipo de injerto. ${ }^{*} \mathrm{Chi}^{2}: \mathrm{p}>0,05$. técnica quirúrgica de colocación del injerto y tamaño de perforación.

Para el cálculo del tamaño de la muestra se utilizó "GPower" y se consideró en un mismo análisis el resultado anatómico y funcional dando un tamaño de muestra total de 81 pacientes con un poder de un $80 \%$. Para el cálculo se consideró un éxito anatómico de $82 \%$ para fascia y de $92 \%$ para cartílago ${ }^{2}$. Para el éxito funcional se consideró $68 \%$ para fascia y $34 \%$ para cartílago ${ }^{1}$. Para el análisis estadístico de las variables se utilizó Chi cuadrado $\left(\mathrm{Chi}^{2}\right)$ y regresión logística.

\section{Resultados}

De un total de 227 fichas, cumplieron criterios de inclusión 154 pacientes. 73 fichas fueron excluidas por mal registro de datos, inasistencia a controles posteriores a cirugía, no contar con audiometrías de control posoperatorio, diagnóstico de otitis media crónica colesteatomatosa o miringoplastía previa. En 102 pacientes $(66 \%)$ se utilizó injerto de fascia de músculo temporal y en 52 pacientes (34\%) injerto de cartílago de trago con o sin pericondrio.

\section{Resultado anatómico según tipo de injerto}

En el grupo en que se utilizó fascia de músculo temporal del total de 102 pacientes, 38 presentaron reperforación del injerto que corresponde a un $37 \%$, mientras que en el grupo de cartílago con o sin pericondrio del total de 52 pacientes, 18 presentaron reperforación del injerto que corresponde a un 35\% (Figura 1). Estos datos se analizaron con $\mathrm{Chi}^{2}$, con un valor $\mathrm{p}$ mayor a 0,05 no encontrándose diferencias estadísticamente significativas entre el tipo de injerto utilizado y resultado anatómico.

\section{Resultado funcional según tipo de injerto}

En el grupo en que se utilizó fascia de músculo temporal, del total de 102 pacientes, 41 presentaron un éxito funcional, lo que corresponde a un $40 \%$ del total. Mientras que en el grupo de cartílago con o sin pericondrio, del total de 52 pacientes, 22 presentaron un éxito funcional que corresponde a un $42 \%$ del total (Figura 2). No hubo diferencias estadísticamente significativas entre el tipo de injerto utilizado y resultado funcional $\left(\mathrm{Chi}^{2}, \mathrm{p}>0,05\right)$. 


\section{Resultado anatómico y tabaquismo}

Del total de 154 pacientes, 38 presentaron el antecedente de tabaquismo activo al momento de la cirugía, de los cuales 20 (53\%) presentaron reperforación del injerto, mientras que 116 no presentaron antecedentes de tabaquismo presentando 36 (31\%) de ellos reperforación del injerto (Figura 3). Para comparar estas distribuciones, los datos se analizaron a través de la prueba de $\mathrm{Chi}^{2}$, observándose diferencias estadísticamente significativas $(\mathrm{p}=0,016)$. Para establecer el valor predictivo positivo del tabaquismo se realizó un análisis inferencial a través de una regresión logística ajustándose por el tamaño de perforación, ya que con otras variables los resultados no fueron significativos. Se estableció que el uso de tabaco predice hasta en un 14,6\% la reperforación del injerto (Tabla 1, $\mathrm{p}=0,001$ ).

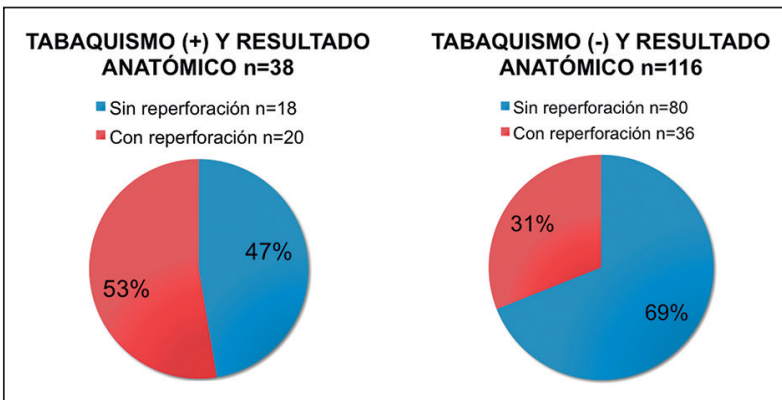

Figura 3. Resultado anatómico y tabaquismo. ${ }^{*} \mathrm{Chi}^{2}: \mathrm{p}<0,05$.

\section{Resultado anatómico y tamaño de perforación (Tabla 2 )}

Perforación menor a 25\%: De un total de 48 pacientes, en 28 se realizó cirugía con fascia de músculo temporal de los cuales 7 presentaron reperforación del injerto equivalente a

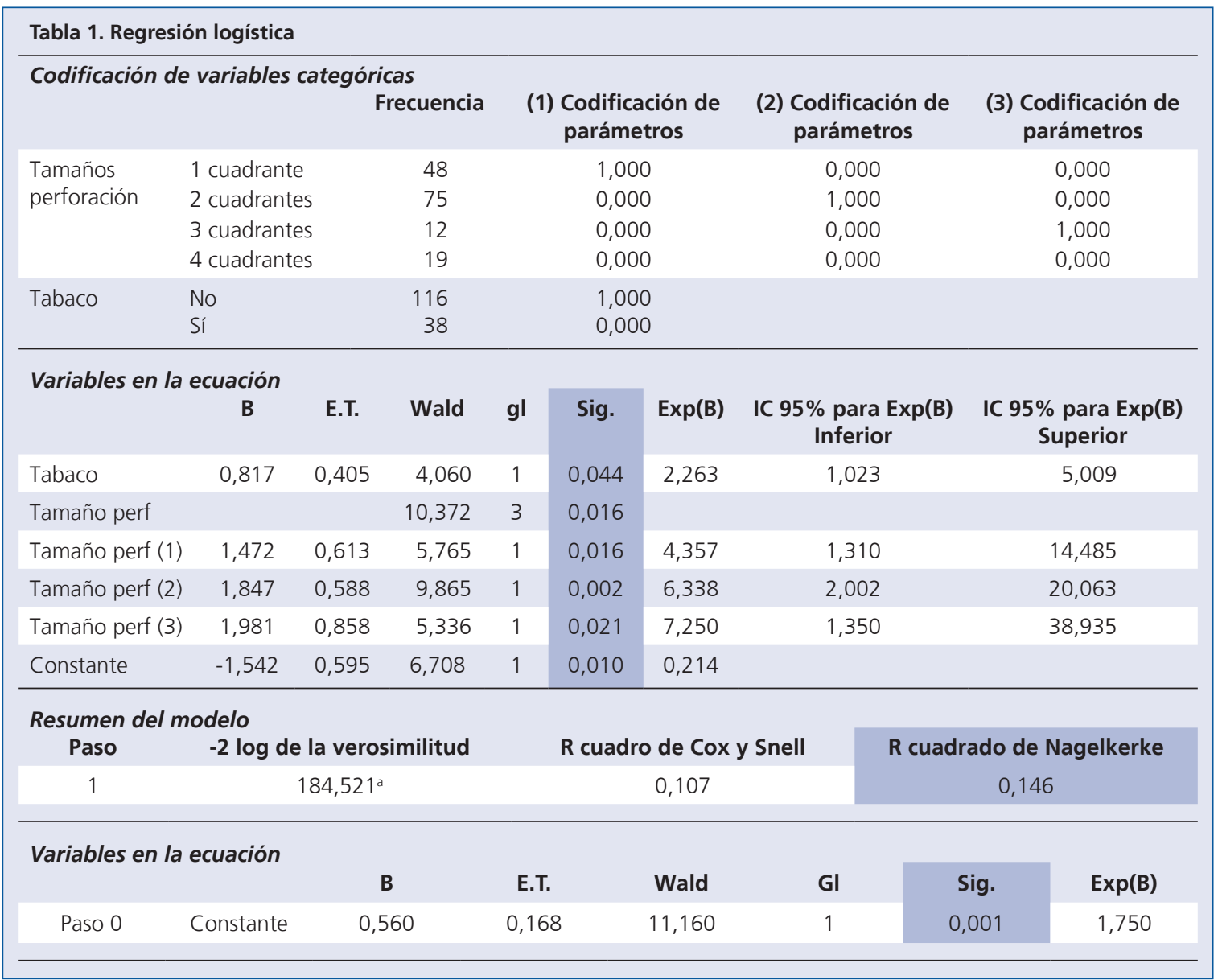


un $25 \%$. Mientras que en 20 se realizó cirugía con cartílago con o sin pericondrio y 10 de ellos presentaron reperforación del injerto equivalente a un $50 \%$.

Perforación 25\% a 50\%: De un total de 75 pacientes, en 51 se realizó cirugía con fascia de músculo temporal y 17 presentaron reperforación del injerto equivalente a un 33,3\%. Mientras que en 24 se utilizó cartílago con o sin pericondrio de los cuales 5 presentaron reperforación del injerto equivalente a un 33,3\%.

Perforación 50\% a 75\%: De un total de 12 pacientes en 8 se realizó cirugía con fascia de músculo temporal, 3 presentaron reperforación del injerto equivalente a un 37,5\%. Mientras que en 4 se utilizó cartílago con o sin pericondrio de los cuales 0 presentaron reperforación del injerto.

Perforación > 75\%: De un total de 19 pacientes en 15 se realizó cirugía con fascia de músculo temporal y 11 presentaron reperforación del injerto equivalente a un $73,3 \%$. Mientras que en 4 se utilizó cartílago con o sin pericondrio de los cuales 3 presentaron reperforación del injerto equivalente a un $75 \%$.

\begin{tabular}{|c|c|c|c|}
\hline $\begin{array}{l}\text { Tamaño } \\
\text { perforación }\end{array}$ & $\begin{array}{l}\text { Sin } \\
\text { reperforación }\end{array}$ & $\begin{array}{c}\text { Con } \\
\text { reperforación }\end{array}$ & Total \\
\hline Menor 25\% & $30(63,8 \%)$ & $17(36,2 \%)$ & 47 \\
\hline $25-50 \%$ & $54(71 \%)$ & $22(29 \%)$ & 76 \\
\hline $50-75 \%$ & $9(75 \%)$ & $3(25 \%)$ & 12 \\
\hline Mayor 75\% & $5(26 \%)$ & $14(74 \%)$ & 19 \\
\hline
\end{tabular}

\begin{tabular}{|c|c|c|c|c|}
\hline $\begin{array}{l}\text { Técnica } \\
\text { quirúrgica }\end{array}$ & repe & $\begin{array}{l}\text { Sin } \\
\text { erforación }\end{array}$ & $\begin{array}{l}\text { Con } \\
\text { reperforación }\end{array}$ & Total \\
\hline Medial (fascia) & 9 & $(64,2 \%)$ & $5(35,7 \%)$ & 14 \\
\hline Lateral (fascia) & 55 & $(62,5 \%)$ & $33(37,5 \%)$ & 88 \\
\hline Isla & 26 & $(66,6 \%)$ & $13(33,3 \%)$ & 39 \\
\hline Empalizada & 7 & $(58,3 \%)$ & $5(41,6 \%)$ & 12 \\
\hline Mariposa & & (100\%) & 0 & 1 \\
\hline
\end{tabular}

Estos datos se analizaron con $\mathrm{Chi}^{2}$ con un valor $\mathrm{p}$ menor a 0,05 encontrándose diferencias estadísticamente significativas entre los diferentes tamaños de perforación y la reperforación del injerto.

\section{Resultado anatómico y tipo de técnica quirúrgica de colocación de injerto (Tabla 3)}

Técnica medial: De un total de 14 pacientes, todos ellos con fascia de músculo temporal, 5 presentaron reperforación del injerto equivalente a un 35,7\%.

Técnica lateral: De un total de 88 pacientes, todos ellos con fascia de músculo temporal, 33 presentaron reperforación del injerto equivalente a un $37,5 \%$.

Técnica en isla: De un total de 38 pacientes, 13 presentaron reperforación del injerto equivalente a un $33,3 \%$.

Técnica en empalizada: De un total de 12 pacientes, 5 presentaron reperforación del injerto equivalente a un $41,6 \%$.

Técnica en mariposa: De un total de 1 paciente, este no presentó reperforación del injerto.

Estos datos se analizaron con $\mathrm{Chi}^{2} \mathrm{p}$ mayor a 0,05 no encontrándose diferencias estadísticamente significativas entre el tipo de injerto y la técnica quirúrgica utilizada respecto al resultado anatómico.

\section{Discusión}

En este estudio no se encontraron mayores diferencias en los resultados anatómicos y audiológicos al comparar injerto de fascia y cartílago como los encontrados en la literatura.

En la literatura se muestra un porcentaje de reperforación menor tanto para fascia (18\%) y cartílago (8\%) en comparación con nuestros resultados fascia $37 \%$ y cartílago $35 \%{ }^{2,8-15}$. Esta diferencia de resultados anatómicos podría ser explicado por la influencia de distintas variables como uso de distintas técnicas, tamaño variable de perforación, diferentes cirujanos que realiza la cirugía, pérdida de pacientes que no se controla después de los 6 meses.

Con relación al resultado funcional, al igual que en la literatura, nuestros resultados no mostraron una diferencia significativa al 
comparar uso de fascia de músculo temporal con cartílago con o sin pericondrio ${ }^{2}$. Al evaluar resultados secundarios se encontró una diferencia estadísticamente significativa de los resultados anatómicos en los pacientes con tabaquismo activo. Se estableció que el uso de tabaco predice hasta en un 14,6\% la reperforación del injerto $(\mathrm{p}=0,001)$. Estos resultados, son comparables a lo descrito en la literatura con una menor probabilidad de un resultado quirúrgico exitoso a corto y largo plazo en comparación con el de un paciente no fumador. Becvarovski y cols. describieron en su trabajo falla del injerto en un $20 \%$ en pacientes no fumadores en comparación a un $60 \%$ en los fumadores ${ }^{5}$.

Este resultado nos permite inferir el efecto negativo del tabaco en la neovascularización y cicatrización del injerto y con ello poder asesorar a nuestros pacientes fumadores a abandonar su uso para poder disminuir el riesgo de reperforación.

$\mathrm{Al}$ analizar el tamaño de la perforación y el injerto utilizado respecto a los resultados anatómicos, se encontró mayor reperforación $(74 \%)$ en perforaciones holotimpánicas, independiente del injerto utilizado (Tabla 2). Esto puede ser dado a que las perforaciones holotimpánicas tienen menos vascularización para fijar el injerto. Los resultados obtenidos son comparables a los encontrados en la literatura. Onal y cols. encontraron en su trabajo retrospectivo de 80 miringoplastías, que en las perforaciones mayores al $50 \%$ el porcentaje de éxito anatómico era de un $45 \%$, mientras que perforaciones menores al 50\% tenían un éxito de un $77,8 \%{ }^{8}$.

Respecto a la técnica quirúrgica de instalación del injerto, en nuestro estudio, las técnicas lateral, medial y en isla tienen un porcentaje de reperforación similar de alrededor de un 35\%, no así la técnica en empalizada que presenta un porcentaje más alto de reperforación (42\%). Esto puede deberse a que se usa preferentemente en perforaciones holotimpánicas, sin embargo, estos datos no son estadísticamente significativos.

En cuanto a las limitaciones de este trabajo, el tipo de estudio dificulta el manejo de las variables que influyen en los resultados de la cirugía. Además, una gran parte de fichas revisadas presentaban datos incompletos por lo que quedaron excluidas. Por lo tanto, sería de mayor utilidad a futuro realizar un estudio prospectivo, randomizado, en el que se analicen ambos tipos de injerto en condiciones similares.

\section{Conclusión}

En este trabajo no se obtuvieron diferencias estadísticamente significativas al analizar los resultados anatómicos y funcionales comparando el uso de injerto de FMT y CT con o sin pericondrio, para el tratamiento quirúrgico de la otitis media crónica simple con miringoplastía. Se encontró un porcentaje mayor de reperforación independiente del tipo de injerto utilizado que lo publicado en la literatura.

Se identificó el tabaco como un factor negativo para el resultado de la miringoplastía, al igual que perforaciones extensas de 4 cuadrantes, independiente del tipo de injerto utilizado. No se encontraron diferencias estadísticamente significativas al analizar el resultado anatómico de las diferentes técnicas utilizadas para colocación de injerto.

\section{Bibliografía}

1. Gómez P, Navarro A, Reyes J, de la Torre Diamante D, Murcia F. Resultados quirúrgicos y funcionales de timpanoplastías tipo I según injerto utilizado. Revista FASO. 2016;3:37-41.

2. Jalali MM, Motasaddi M, Kouhi A, Dabiri S, Soleimani R. Comparison of cartilage with temporalis fascia tympanoplasty: A meta-analysis of comparative studies. Laryngoscope. 2017;127(9):2139-2148. doi: 10.1002/lary.26451.

3. Kageyama-Escobar AM, Rivera-Moreno MA, RiveraMéndez A. Factores de riesgo en el fracaso de la miringoplastía. Academia Nacional de Medicina de México. 2001;137(3):209-220.

4. Zelada U, Arregui R, Palacios E. Timpanoplastía en niños. Experiencia de 10 años. Rev Otorrinolaringol Cir Cabeza Cuello. 2005; 65:92-96.

5. Becvarovski Z, Kartush JM. Smoking and tympanoplasty: implications for prognosis and the Middle Ear Risk Index (MERI). Laryngoscope. 2001;111(10):1806-1811. doi: 10.1097/00005537200110000-00026

6. Yang T, Wu X, Peng X, Zhang Y, Xie S, Sun H. Comparison of cartilage graft and fascia in type 1 tympanoplasty: systematic review and meta- 


\section{ARTÍCULO DE INVESTIGACIÓN}

analysis. Acta Otolaryngol. 2016;136(11):1085-1090. doi: 10.1080/00016489.2016.1195013

7. Mohamad SH, Khan I, Hussain SS. Is cartilage tympanoplasty more effective than fascia tympanoplasty? A systematic review. Otol Neurotol. 2012;33(5):699-705. doi: 10.1097/ MAO.0b013e318254fbc2

8. Onal K, Uguz MZ, Kazikdas KC, Gursoy ST, Gokce H. A multivariate analysis of otological, surgical and patient-related factors in determining success in myringoplasty. Clin Otolaryngol. 2005;30(2):115-120. doi: 10.1111/j.1365-2273.2004.00947.x

9. Demirci S, Tuzuner A, Karadas H, Acıkgoz C, Caylan $\mathrm{R}$, Samim EE. Comparison of temporal muscle fascia and cartilage grafts in pediatric tympanoplasties. Am J Otolaryngol. 2014;35(6):796-799. doi: 10.1016/j. amjoto.2014.07.011

10. Otte-García J. et al. Investigación de algunos problemas de las OMC en Chile. Rev Otorrinolaringología Cir Cabeza Cuello. 1973;33:1-21.
11. Plaza E, Otte-García J. Análisis crítico de timpanoplastías. Rev Otorrinolaringología Cir Cabeza Cuello. 1980;40:6-11.

12. Esquivel P, Naser A, Bustamante C. Timpanoplastía en niños. Experiencia de 10 años en el Hospital Clínico de la Universidad de Chile. Rev Otorrinolaringol Cir Cabeza Cuello. 2006;66:7-12.

13. Caro J, Glasinovic A, Dueñas R, Elissalde L. Análisis de 168 timpanoplastías. Rev Otorrinolaringología Cir Cabeza Cuello. 1983;43:17-23.

14. Nicklas L, Salgado S, Arancibia M, Orellana JJ. Timpanoplastías en pacientes mayores de 50 años. Experiencia en el Hospital San Juan de Dios. Rev Otorrinolaringología Cir Cabeza Cuello. 2007;67:2630.

15. Toro C, Naser A, Sanhueza C, Valdés C, Gormaz JP, Molina V, Leslie A. Timpanoplastía en adultos en el Hospital Clínico de la Universidad de Chile: Revisión de 10 años. Rev Otorrinolaringología Cir Cabeza Cuello. 2007;67:237-243. 